International Journal of Pure and Applied Mathematics

Volume 90 No. 1 2014, 11-24

ISSN: 1311-8080 (printed version); ISSN: 1314-3395 (on-line version)

url: http://www.ijpam.eu

doi: http://dx.doi.org/10.12732/ijpam.v90i1.3

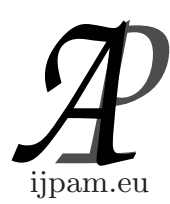

\title{
PERISTALTIC TRANSPORT OF A CASSON FLUID \\ IN A CHANNEL WITH PERMEABLE WALLS
}

\author{
A.N.S. Srinivas ${ }^{1 \S}$, R. Hemadri Reddy ${ }^{2}$, S. Srinivas ${ }^{3}$, S. Sreenadh ${ }^{4}$ \\ ${ }^{1,2,3}$ School of Advanced Sciences \\ VIT University \\ Vellore, Tamil Nadu, 632 014, INDIA \\ ${ }^{4}$ Department of Mathematics \\ Sri Venkateswara University \\ Tirupati, 517 502, INIDA
}

\begin{abstract}
Peristaltic transport of a Casson fluid in a channel with permeable walls is investigated. Exact analytical solution of the flow quantities are developed under long wavelength and low Reynolds number assumptions. The effects of Darcy number and yield stress on the pumping characteristics are obtained and discussed. It is observed that for a given $\Delta P$, the flux $\bar{Q}$ decreases with increasing Darcy number. Further, it is noticed that for a given $\Delta P$, the flux $\bar{Q}$ decreases with increasing yield stress $\tau$. This may be due to the yield stress behaviour of the Casson fluid. The results of the trapping phenomenon are analyzed. Four different wave forms are considered. The comparison of the different wave forms is discussed.
\end{abstract}

Key Words: peristalsis, Casson fluid, channel flow, permeable walls, wave forms

\section{Introduction}

Peristalsis is an important mechanism reported in many organisms and in a va-

Received: July 31, 2013

(c) 2014 Academic Publications, Ltd.

$\S_{\text {Correspondence author }}$ url: www.acadpubl.eu 
riety of organs of a living body. Peristaltic flows also provide efficient means for sanitary fluid transport and are thus exploited in industrial peristaltic pumping and medical devices. This mechanism is mainly used in the mechanical roller pumps using viscous fluids in the printing industry and for transporting blood in heart lung machine. Raju and Devanathan (1972) were the first authors to investigate peristaltic motion of a non-Newtonian fluid quantitatively. Srivastava and Srivastava (1994) discussed the problem of peristaltic transport of blood assuming a single layer. Later, Srivastava (1987) considered the ax symmetric flow of Casson fluid in a circular non-uniform tube. A mathematical model for peristaltic motion of a non-linear viscous flow was proposed by Tang and Rankin (1993) and they used an iterative method to solve a true boundary problem. Peristaltic motion of a generalized Newtonian fluid in a non-uniform channel is studied by Elshehaway (1998). Peristaltic motion of an incompressible generalized Newtonian fluid in a planar channel is analyzed by El misery et.al. (1996). Mishra and Ghosh (1997) modeled the smallest microscopic blood vessels in human lungs as sheet like networks and each sheet is idealized as a channel bounded by two thin layers of porous media. They found the velocity and pressure distribution in the channel and discussed the results numerically.

Scott Blair (1959) observed that the Casson model was well suitable for the discussion of blood flow. Chein and Kurland (1965) discussed viscometry of human blood using Cassons equation. In Cassons equation the yield stress of the non-Newtonian fluid is taken into consideration. Herschel-Bulkley model is another of the yield stress model, which also describes the blood flow in small vessels. Using this model Vajravelu et al. (2005a, 2005b, 2006) made investigations on peristaltic pumping of Herschel-Bulkley fluid in a channel with flexible wall. Hariharan et.al, (2008) have investigated the Peristaltic transport of a Non-Newtonian fluid in a diverging tube with different waveforms. Hayat et al. (2007) investigated peristaltic flow of a micropolar fluid in a channel with different wave forms and Hayat et al. (2008) have investigated the MHD peristaltic channel flow of a Jeffrey fluid with complaint walls with porous medium.

All these investigations deal with peristaltic pumping of biofluids in ducts with impermeable walls. Among several biofluid flows in psychological systems, blood flow in small blood vessels is reported to flow under the mechanism of peristalsis. In view of this a better understanding of the peristaltic pumping in blood vessels can be made, if we idealize the blood vessel as a channel with permeable walls. In this paper peristaltic pumping of a Casson fluid in a channel with permeable walls is investigated under long wavelength and low Reynolds number assumptions. The expressions for velocity field, the stream function 
and the pressure rise are derived. The effect of Darcy number on the pumping characteristics is discussed.

\section{Mathematical Formulation and Solution}

Consider the peristaltic transport of a Casson fluid in a channel with permeable wall of half-width ' $a$ '. A longitudinal train of progressive sinusoidal waves takes place on the upper and lower walls of the channel. For simplicity, we restrict our discussion to the half width of the channel as shown in figure (1). The region between $y=0$ and $y=y_{0}$ is called plug flow region. In the plug flow region $\left|\tau_{x y}\right| \leq \tau_{0}$, in the region $y=y_{0}$ to $y=h,\left|\tau_{x y}\right|>\tau_{0}$.

The wall deformation is given by

$$
H=a+b \operatorname{Sin} \frac{2 \pi}{\lambda}(X-c t)
$$

where $b$ is the amplitude, $\lambda$ is the wavelength and $c$ is the wave speed.

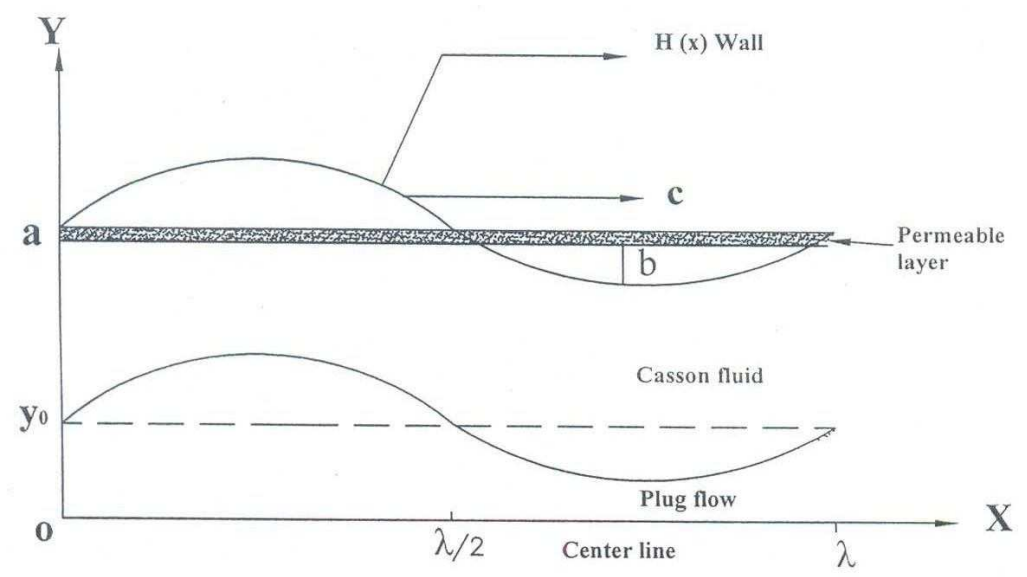

Figure 1: Physical Model

\subsection{Equations of Motion}

Under the assumptions that the channel length is an integral multiple of the wavelength $\lambda$ and the pressure difference across the ends of the channel is a constant, the flow becomes steady in the wave frame $(x, y)$ moving with velocity 
c away from the fixed (laboratory) frame $(X, Y)$. The transformation between these two frames is given by

$$
x=X-c t ; y=Y ; u(x, y)=U(X-c t, Y)-c ; v(x, y)=V(X-c t, Y)
$$

Using the non-dimensional quantities

$$
\begin{aligned}
& \bar{u}=\frac{u}{c} ; \bar{x}=\frac{x}{\lambda} ; \bar{y}=\frac{y}{a} ; \bar{h}=\frac{h}{a} ; \bar{t}=\frac{c t}{\lambda} \\
& \bar{\psi}=\frac{\psi}{a c} ; \bar{p}=\frac{p a^{2}}{\lambda \mu c} ; \bar{q}=\frac{q}{a c} ; \varphi=\frac{b}{a} ; \quad D a=\frac{k}{a^{2}}
\end{aligned}
$$

The non-dimensional form of equation governing the motion (dropping the bars) is

$$
\frac{\partial}{\partial y}\left[-\frac{\partial u}{\partial y}+\tau_{0}+2 \sqrt{-\tau_{0} \frac{\partial u}{\partial y}}\right]=-\frac{\partial p}{\partial x}, \quad 0 \leq y \leq h
$$

The non-dimensional boundary conditions are

$$
\begin{gathered}
\frac{\partial u}{\partial y}=0 \text { at } y=0 \\
u=-1-\frac{\sqrt{D a}}{\alpha} \frac{\partial u}{\partial y} \text { at } y=h
\end{gathered}
$$

(Saffman slip condition, 1971)

\subsection{Solution}

Solving equation (4) by using the boundary conditions (5) and (6) we get the velocity as

$$
\begin{aligned}
u=-1 & -\frac{P}{2}\left[y^{2}-h^{2}-2 h \frac{\sqrt{D a}}{\alpha}\right]-2 \tau_{0}\left[y-h-\frac{\sqrt{D a}}{\alpha}\right] \\
+ & \frac{4 \sqrt{\tau_{0}}}{3 P}\left[\left(P y+\tau_{0}\right)^{\frac{3}{2}}-\left(P h+\tau_{0}\right)^{\frac{3}{2}}\right]-2 \sqrt{\tau_{0}} \frac{\sqrt{D a}}{\alpha}\left(P h+\tau_{0}\right)^{\frac{1}{2}} \\
u_{p}=-1- & \frac{P}{2}\left[y_{0}^{2}-h^{2}-2 h \frac{\sqrt{D a}}{\alpha}\right]-2 \tau_{0}\left[y_{0}-h-\frac{\sqrt{D a}}{\alpha}\right] \\
+ & \frac{4 \sqrt{\tau_{0}}}{3 P}\left[\left(P y_{0}+\tau_{0}\right)^{\frac{3}{2}}-\left(P h+\tau_{0}\right)^{\frac{3}{2}}\right]-2 \sqrt{\tau_{0}} \frac{\sqrt{D a}}{\alpha}\left(P h+\tau_{0}\right)^{\frac{1}{2}}
\end{aligned}
$$




$$
0 \leq y \leq y_{0}
$$

Integrating the equation (7) and (8), we obtain the stream function as

$$
\begin{aligned}
\psi=-y & -\frac{P}{2}\left[\frac{y^{3}}{3}-h^{2} y-2 h \frac{\sqrt{D a}}{\alpha} y+\frac{2 y_{0}^{3}}{3}\right]-2 \tau_{0}\left[\frac{y^{2}}{2}-h y-\frac{\sqrt{D a}}{\alpha} y+\frac{y_{0}^{2}}{2}\right] \\
& +\frac{4 \sqrt{\tau_{0}}}{3 P}\left[\frac{2\left(P y+\tau_{0}\right)}{5 P}-y\left(P h+\tau_{0}\right)^{\frac{3}{2}}-\frac{2\left(P y_{0}+\tau_{0}\right)^{5 / 2}}{5 P}+y_{0}\left(P y_{0}+\tau_{0}\right)^{3 / 2}\right] \\
& -2 y \sqrt{\tau_{0}} \frac{\sqrt{D a}}{\alpha}\left(P h+\tau_{0}\right)^{\frac{1}{2}}
\end{aligned}
$$

and

$$
\begin{aligned}
\psi_{p}=-y_{0} & -\frac{P}{2}\left[y_{0}{ }^{3}-h^{2} y_{0}-2 h \frac{\sqrt{D a}}{\alpha} y_{0}\right]-2 \tau_{0}\left[y_{0}^{2}-h y_{0}-\frac{\sqrt{D a}}{\alpha} y_{0}\right] \\
+ & \frac{4 \sqrt{\tau_{0}}}{3 P}\left[y_{0}\left(P y_{0}+\tau_{0}\right)^{\frac{3}{2}}-y_{0}\left(P h+\tau_{0}\right)^{\frac{3}{2}}\right]-2 y_{0} \sqrt{\tau_{0}} \frac{\sqrt{D a}}{\alpha}\left(P h+\tau_{0}\right)^{\frac{1}{2}}
\end{aligned}
$$

The volume flux through each cross section in the wave frame is given by

$$
\begin{aligned}
q= & \int_{0}^{y_{0}} u_{p} d y+\int_{y_{0}}^{h} u d y \\
= & \frac{P}{3}\left[h^{3}-y_{0}^{3}+3 h^{2} \frac{\sqrt{D a}}{\alpha}\right]+\tau_{0}\left[h^{2}-y_{0}^{2}+\frac{h}{2} \frac{\sqrt{D a}}{\alpha}\right] \\
& +\frac{4 \sqrt{\tau_{0}}}{3 P}\left\{y_{0}\left(P y_{0}+\tau_{0}\right)^{\frac{3}{2}}-h\left(P h+\tau_{0}\right)^{\frac{3}{2}}+\frac{2}{5 P}\left[\left(P h+\tau_{0}\right)^{\frac{5}{2}}-\left(P y_{0}+\tau_{0}\right)^{\frac{5}{2}}\right]\right\} \\
& -2 \tau_{0} h \frac{\sqrt{D a}}{\alpha}\left(P h+\tau_{0}\right)^{\frac{1}{2}}-h
\end{aligned}
$$

The instantaneous volume flow rate $Q(X, t)$ in the laboratory frame between the centre line and the wall is

$$
\begin{aligned}
& Q(X, t)=\int_{0}^{H} U(X, y, t) d y \\
& =\frac{P}{3}\left[h^{3}-y_{0}^{3}+3 h^{2} \frac{\sqrt{D a}}{\alpha}\right]+\tau_{0}\left[h^{2}-y_{0}^{2}+\frac{h}{2} \frac{\sqrt{D a}}{\alpha}\right]
\end{aligned}
$$




$$
\begin{aligned}
& +\frac{4 \sqrt{\tau_{0}}}{3 P}\left\{y_{0}\left(P y_{0}+\tau_{0}\right)^{\frac{3}{2}}-h\left(P h+\tau_{0}\right)^{\frac{3}{2}}+\frac{2}{5 P}\left[\left(P h+\tau_{0}\right)^{\frac{5}{2}}-\left(P y_{0}+\tau_{0}\right)^{\frac{5}{2}}\right]\right\} \\
& \quad-2 \tau_{0} h \frac{\sqrt{D a}}{\alpha}\left(P h+\tau_{0}\right)^{\frac{1}{2}}
\end{aligned}
$$

From equation (11) we have to fix $\mathrm{P}$ in order to calculate the pressure rise $\Delta P$. Since right hand side of equation (11) is non-linear, we assume that the powers of $\tau_{0}$, which are greater than 1 are negligible. In view of this approximation we obtain the expression for $\frac{d p}{d x}$ from equation (11) as

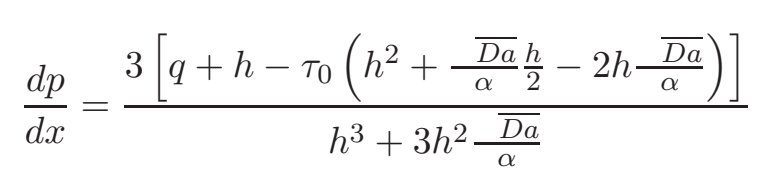

The dimensionless average volume flow rate $\bar{Q}$ over one wavelength is obtained as

$$
\bar{Q}=\frac{1}{T} \int_{0}^{T} Q d t=q+1
$$

\subsection{The Pumping Characteristics}

Integrating the equation (13) with respect to $\mathrm{x}$ over one wavelength, we get the pressure rise (drop) over one cycle of the wave as

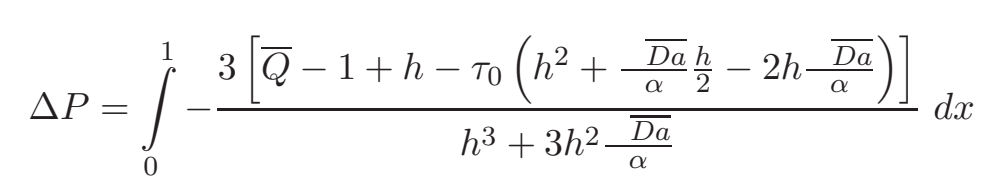

The pressure rise required to produce zero average flow rate is denoted by $\Delta P_{0}$. Hence $\Delta P_{0}$ is given by

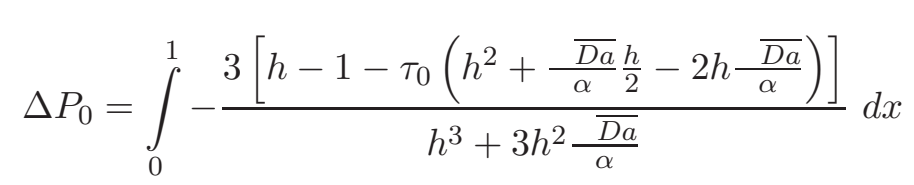

The dimensionless frictional force $F$ at the wall across one wavelength is given by

$$
F=\int_{0}^{1} h\left(-\frac{d p}{d x}\right) d x
$$




$$
=\int_{0}^{1}-\frac{3\left[\bar{Q}-1+h-\tau_{0}\left(h^{2}+\frac{\sqrt{ } \overline{D a}}{\alpha} \frac{h}{2}-2 h \frac{\sqrt{ } \overline{\overline{D a}}}{\alpha}\right)\right]}{h^{2}+3 h \frac{\sqrt{\overline{D a}}}{\alpha}} d x
$$

\section{Discussion of the Results}

From equation (15), we have calculated the pressure difference as a function of $\bar{Q}$ for different values of Darcy number $D a$, for fixed $\varphi=0.4, \tau_{0}=0.1$ and $\alpha=0.2$ is shown in figure (2), we observe that for a given $\Delta P$, the flux $\bar{Q}$ decreases with increasing Darcy number. This is because increasing the Darcy number gives rise to an increase in the permeability of the wall and hence the flux decreases. For a given flux $\bar{Q}$, the pressure difference $\Delta P$ decreases with increase in Darcy number. For free pumping, the flux decreases with increasing Darcy number $D a$. The variation of pressure rise $\Delta P$ with time averaged flow rate is calculated from equation (15) for different values of slip parameter $\alpha$ and is shown in figure (3), for fixed $D a=0.01, \tau_{0}=0.1$ and $\varphi=0.4$. We observe that the longer the value of the slip parameter, the greater the larger pressure rise against which the pump works. For a given $\Delta P$, the flux $\bar{Q}$ increases with the increasing $\alpha$. For a given flux $\bar{Q}$, the pressure rise depends on $\alpha$ and it increases with increasing $\alpha$.

From equation (17), we have calculated the pressure difference as a function of $\bar{Q}$ for different values of amplitude ratios $\varphi$, for fixed $D a=0.01, \tau_{0}=0.1$ and $\alpha=0.2$ is shown in figure (4). One can observe that the larger the amplitude ratio, the greater the pressure rise against which the pump works. For a given flux $\bar{Q}$, the pressure difference $\Delta P$ increases with increasing $\varphi$. For a given $\Delta P$, the flux $\bar{Q}$ increases with increasing $\varphi$.

The variation of pressure rise with time averaged flow rate is calculated from equation (15) for different values of yield stress $\tau_{0}$ and is shown in figure (5) for fixed $D a=0.01, \alpha=0.2$ and $\varphi=0.4$. We observe that for a given $\Delta P$ the flux $\bar{Q}$ decreases with increasing yield stress $\tau_{0}$. This may be due to the yield stress behaviour of the Casson fluid. For a given flux $\bar{Q}$, the pressure difference $\Delta P$ depends on yield stress $\tau_{0}$ and it decreases with increasing $\tau_{0}$. For free pumping $(\Delta P=0)$ the flux $\bar{Q}$ decreases with increasing yield stress.

From equation (15), we have calculated frictional force as a function of $\bar{Q}$, for various values of $D a, \varphi, \tau_{0}$ and $\alpha$ is shown in figures (6) to (9). It is noticed 
that the frictional force $\mathrm{F}$ shows opposite behaviour compared to pressure rise $\Delta P$. As the yield stress $\tau_{0}$ increases, the flux $\bar{Q}$ decreases for a given frictional force.

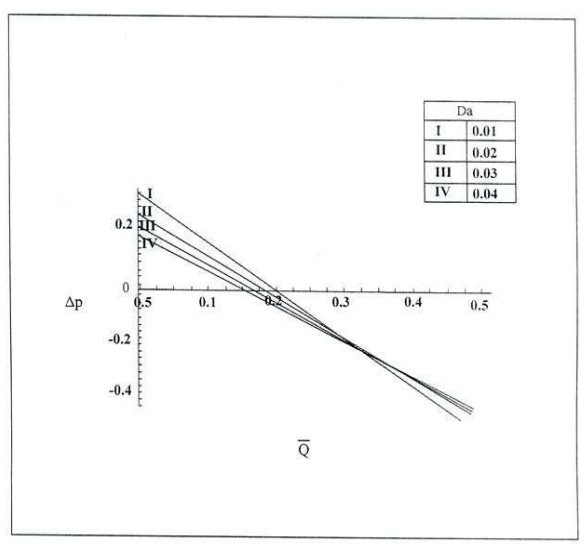

Figure 2: The variation of $\Delta p$ with for different values of $D a$ with $\phi=$ $0.4, \tau_{0}=0.1$ and $\alpha=0.2$

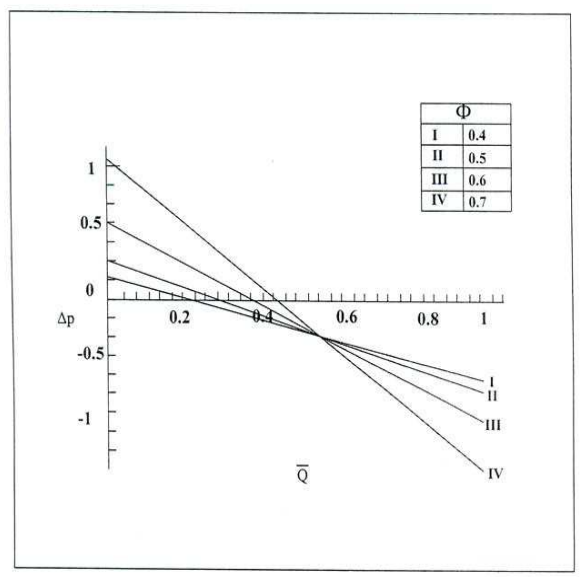

Figure 4: The variation of $\Delta p$ with for different values of $D a$ with $\phi=$ $0.4, \tau_{0}=0.1$ and $\alpha=0.2$

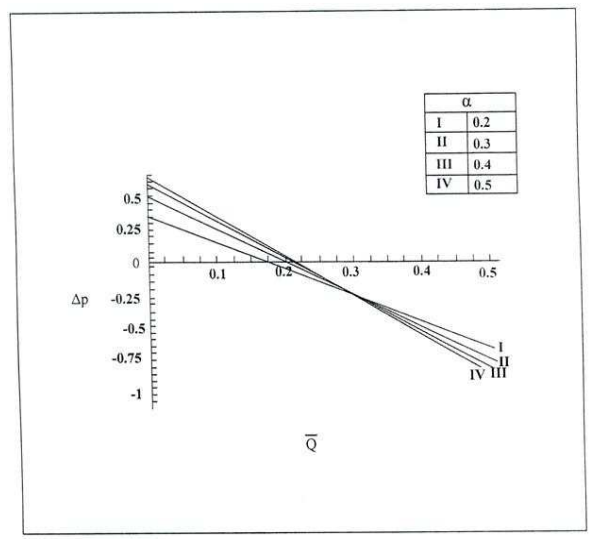

Figure 3: The variation of $\Delta p$ with for different values of $D a$ with $\phi=$ $0.4, \tau_{0}=0.1$ and $\alpha=0.2$

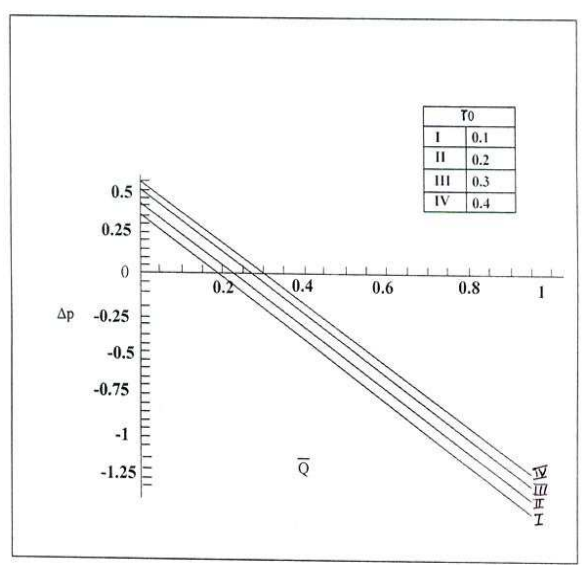

Figure 5: The variation of $\Delta p$ with for different values of $D a$ with $\phi=$ $0.4, \tau_{0}=0.1$ and $\alpha=0.2$ 


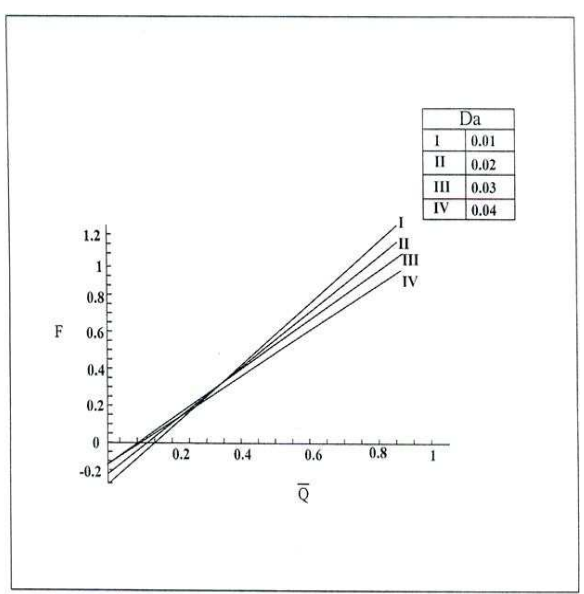

Figure 6: The variation of $\Delta p$ with for different values of $D a$ with $\phi=$ $0.4, \tau_{0}=0.1$ and $\alpha=0.2$

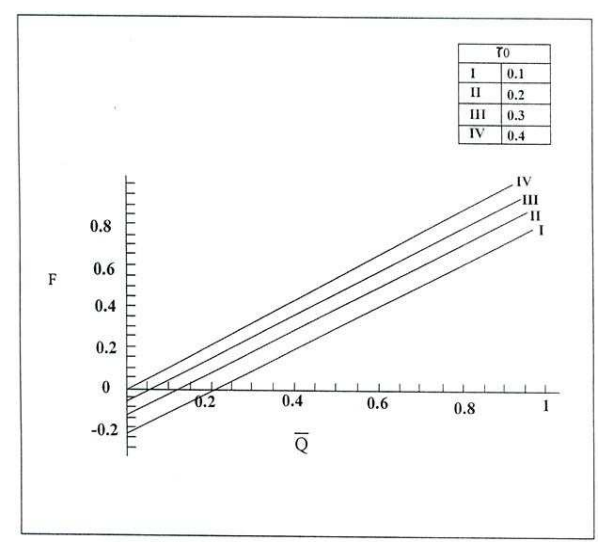

Figure 8: The variation of $\Delta p$ with for different values of $D a$ with $\phi=$ $0.4, \tau_{0}=0.1$ and $\alpha=0.2$

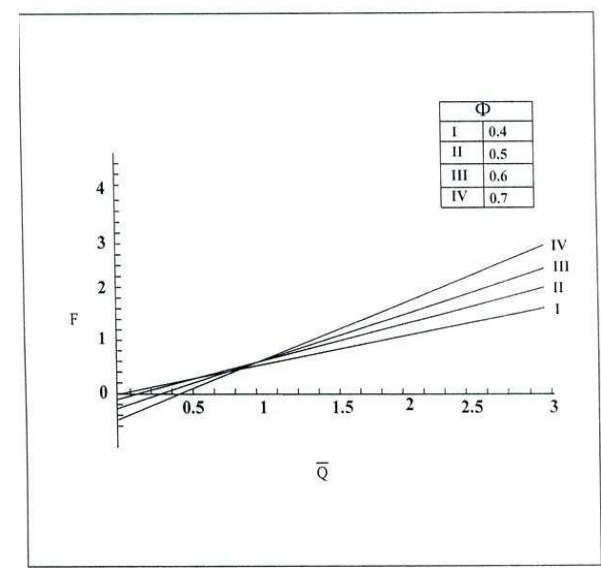

Figure 7: The variation of $\Delta p$ with for different values of $D a$ with $\phi=$ $0.4, \tau_{0}=0.1$ and $\alpha=0.2$



Figure 9: The variation of $\Delta p$ with for different values of $D a$ with $\phi=$ $0.4, \tau_{0}=0.1$ and $\alpha=0.2$

\section{Expression for the Wave Shape}

The non-dimensional expressions for the four different wave forms are given by the following 

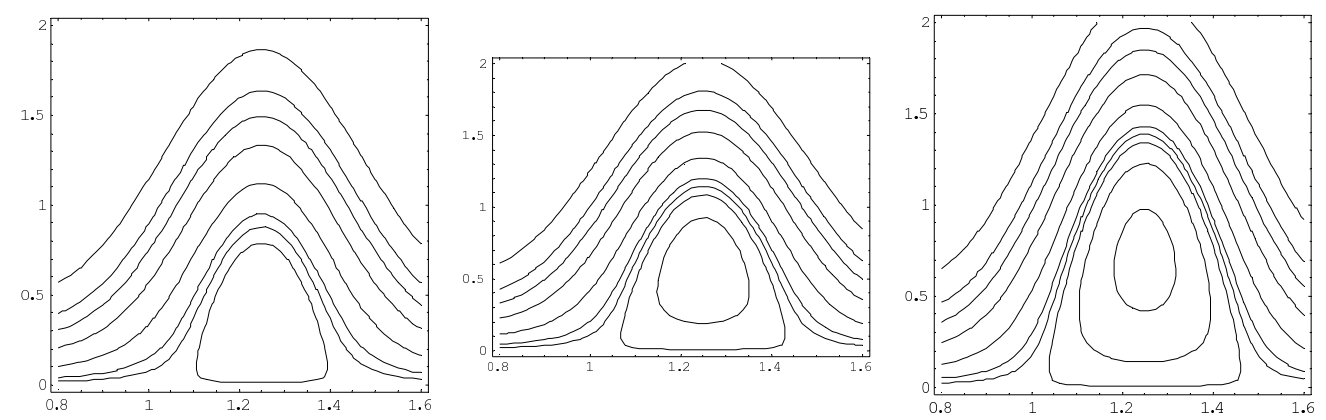

Figure 10: Streamlines for different values of Darcy number (i) Da=15 (ii) $\mathrm{Da}=18$ (iii) $\mathrm{Da}=21$ with $\tau_{0}=0.1, \phi=0.6, Q=0.6, \alpha=0.3$ and $y_{0}=0.1$
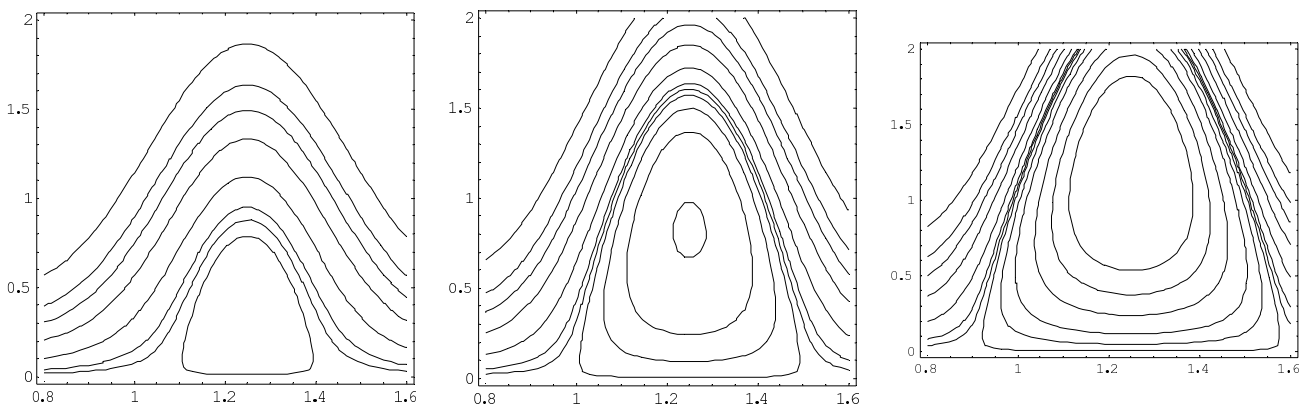

Figure 11: Streamlines for different values of (i) $\tau_{0}=0.1$ (ii) $\tau_{0}=0.12$ (iii) $\tau_{0}=0.15$ with $D a=15, \phi=0.6, Q=0.6, \alpha=0.3$ and $y_{0}=0.1$

1. Sinusoidal wave:

$$
h(x, t)=1+\varphi \operatorname{Sin}[2 \pi x]
$$

2. Triangular wave:

$$
h(x, t)=1+\varphi\left[\frac{8}{\pi^{3}} \sum_{m=1}^{\infty} \frac{(-1)^{m+1}}{(2 m-1)^{2}} \operatorname{Sin}\{2(2 m-1) \pi x\}\right]
$$

3. Square wave:

$$
h(x, t)=1+\varphi\left[\frac{4}{\pi} \sum_{m=1}^{\infty} \frac{(-1)^{m+1}}{(2 m-1)} \operatorname{Cos}\{2(2 m-1) \pi x\}\right]
$$



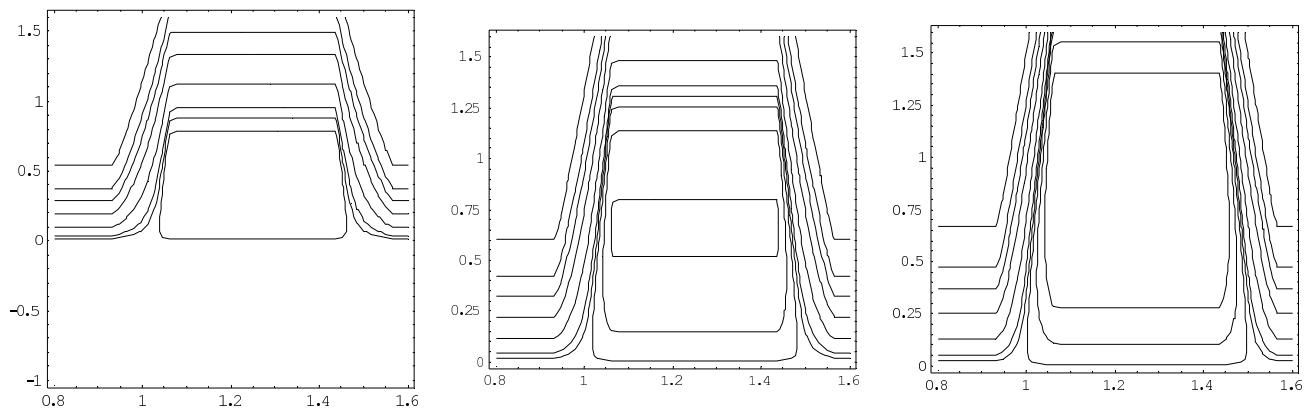

Figure 12: Streamlines for different values of Darcy number (i) Da=15 (ii) $\mathrm{Da}=20$ (iii) $\mathrm{Da}=25$ with $\tau_{0}=0.1, \phi=0.6, Q=0.6, \alpha=0.3$ and $y_{0}=0.1$
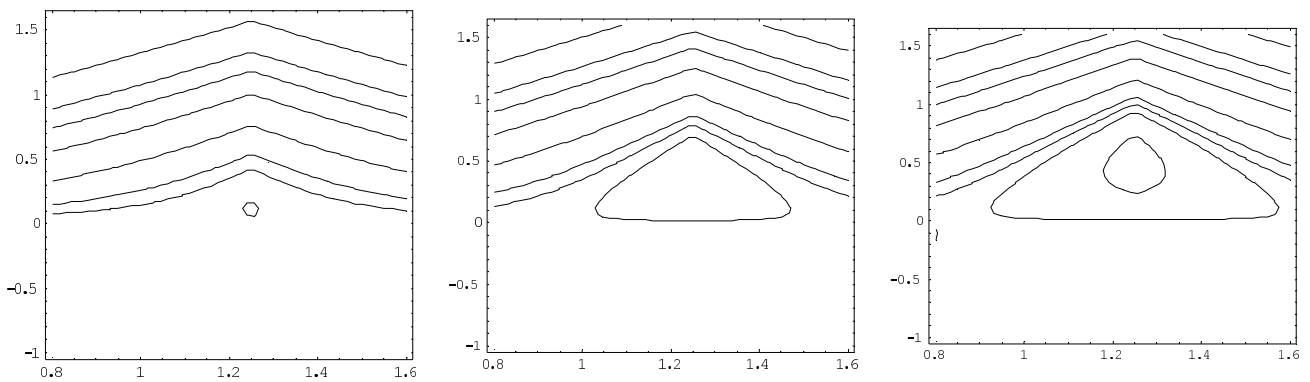

Figure 13: Streamlines for different values of Darcy number (i) $\mathrm{Da}=20$ (ii) $\mathrm{Da}=25$ (iii) $\mathrm{Da}=28$ with $\tau_{0}=0.1, \phi=0.6, Q=0.6, \alpha=$ 0.3 and $y_{0}=0.1$

4. Trapezoidal wave:

$$
h(x, t)=1+\varphi\left[\frac{32}{\pi^{2}} \sum_{m=1}^{\infty} \frac{\operatorname{Sin} \frac{\pi}{8}(2 m-1)}{(2 m-1)^{2}} \operatorname{Sin}\{2(2 m-1) \pi x\}\right]
$$

Note that the expressions for the triangular, square and trapezoidal waves are derived from the Fourier series.

\section{Trapping Phenomenon}

Another important phenomenon in peristaltic transport is trapping. The formation of an internally circulating bolus of fluid by closed streamlines is called 

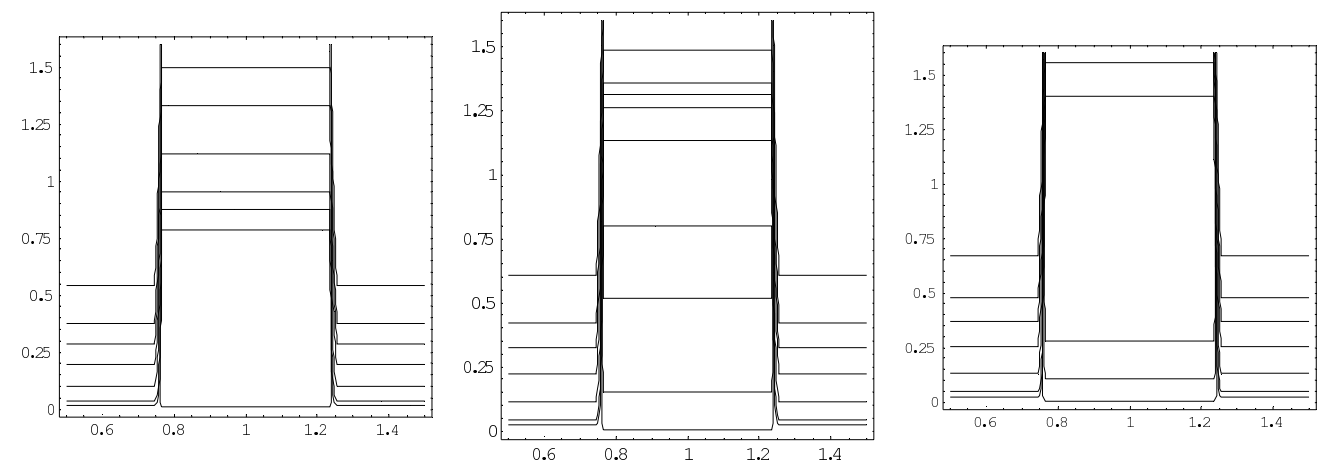

Figure 14: Streamlines for different values of Darcy number (i) Da=15 (ii) $\mathrm{Da}=20$ (iii) $\mathrm{Da}=25$ with $\tau_{0}=0.1, \phi=0.6, Q=0.6, \alpha=$ 0.3 and $y_{0}=0.1$

trapping and is pushed a head along with the peristaltic wave. The physical phenomena may be responsible for thrombus formation in blood and the movement of flood bolus in gastrointestinal tract. The streamlines in the wave frame for fixed values of $\varphi=0.6, Q=0.6, \alpha=0.3$ and $y_{0}=0.1$ and for different values of $D a$ and $\tau_{0}$ are discussed. From the fig (10) the streamlines in the wave frame for pumping with $\varphi=0.6, Q=0.6, \alpha=0.3$ and $y_{0}=0.1$ for different values of $D a$. It is observed from the figure that the size of the trapping zone is decreasing with increasing $D a$. The streamlines in the wave frame, for pumping with $\varphi=0.6, Q=0.6, \alpha=0.3$ and $y_{0}=0.1$ for different values $\tau_{0}$. It is seen that the trapping zone is increases with increasing $\tau_{0}$. For different wave forms, when the Darcy number increases the size of the trapped bolus increases but in triangular wave at $D a=15$ no bolus was forming. The effect of $D a$ for different waveforms can also be seen through graphs from fig. 12-14.

\section{References}

[1] Casson.N (1959). Rheology of Disperse systems, Edited by C. C. Mill, Pergamon Press, New York.

[2] Das, B. and R.L Batra (1993). Secondary flow of a Casson fluid in a slightly curved tube, Int. J. Non-linear Mechanics, 28:5, 567-577.

[3] EL Misery, A.M.Elsheawey and A.Hakeem (1996). Peristaltic motion of an incompressible generalized Newtonian fluid in a planar channel, J. Phys. Soc.Japan, 65:11, 3524-3529. 
[4] EL Shehawey. E.F, and S.Z.A Hussen (2000) Effects of porous boundaries on peristaltic transport through a porous medium, Acta Mechanica, 143, 165-177.

[5] EL Shehawey. E.F, and EL Sebaei, W (2000). Peristaltic transport in a cylindrical tube through a porous medium, Int.J. Math. Math. Sci., 24, 217-230.

[6] Hayat.T, N.Ali, and Z.Abbas (2007). Peristaltic flow of a micropolar fluid in a channel with different wave forms, Physics letters - A, 370, 331-334.

[7] Hayat.T, M.Javad, N.Ali, (2008). MHD peristaltic Transport of a Jeffery Fluid in a channel with compliant walls and porous space, Transp.Porous Med. 74, 259-274.

[8] Hariharan.P, R.Seshadri, R.K.Banerjee (2008). Peristaltic transport of Non-Newtonian fluid in a diverging tube with different waveforms, Math. Comput.Model. 48, 998-1017.

[9] Kothandapani.M and S.Srinivas (2008). Non-linear peristaltic transport of a Newtonian fluid an inclined asymmetrical channel through porous medium, Physics Letters-A, 372, 1265-1276.

[10] Mishra .J.C and S.K.Ghosh (1997). A mathematical model for the study of blood flow through a channel with permeable walls, Acta mechanica, $122,137-153$.

[11] Nagarani.P (2010). Peristaltic transport of a Casson fluid in an inclined channel, Korea Australia Rheology Journal, 22 :2, 105-111.

[12] Raju, K.K. and R.Devanathan (1972). Peristaltic motion of a nonNewtonian fluid part I, Rheol. Acta. 11,170-178.

[13] Raju, K.K. and R.Devanathan (1974). Peristaltic motion of a nonNewtonian fluid part-II : Visco-elastic fluid, Rheol. Acta, 13, 944-948.

[14] Scott Blair, G.W (1959). An equation for the flow of blood plasma and serum through glass capillaries, Nature 183, 613-614.

[15] Siddiqui, A.M., A.Provost and W.H.Schwarz (1991). Peristaltic pumping of a second-order fluid in a planar channel, Rheol.Acta, 30, 249-262. 
[16] Srinivas.S and R.Gayathri (2009). Peristaltic transport of a Newtonian fluid in a vertical asymmetric channel with heat transfer and porous medium, Applied Mathematics and Computation, 215, 185-196.

[17] Srivastava.L.M. and V.P.Srivastava (1984). Peristaltic transport of blood: Casson model II, J.Biomech., 17, 821-829.

[18] Tang, D. and V.Rankin (1993). Numerical and asymptotic solutions for peristaltic motion of nonlinear viscous flows with elastic free boundaries, Siam J.Sci.Comput.,14:6,1300-1319.

[19] Vajravelu. K., S.Sreenadh and V.R. Babu (2005). Peristaltic pumping of a Herschel-Bulkley fluid in a channel, Appl. Math. and Computation 169, 726-735.

[20] Vajravelu.K, S.Sreenadh and V.R.Babu (2005). Peristaltic pumping of a Herschel-Bulkley fluid in an inclined tube, Int. J. Non-linear Mech. 40, 83-90.

[21] Vajravelu.K, S.Sreenadh and V.R.Babu (2006). Peristaltic pumping of a Herschel- Bulkley fluid in contact with a Newtonian fluid, Quarterly of Appl. Math. 64, 593-604. 